Paper accepted for publication in Annals of Behavioral Medicine (January 13, 2016). The final publication is available at Springer via http://dx.doi.org/10.1007/s12160-016-9776-x

\title{
Mind the Gap? An Intensive Longitudinal Study of Between-Person and Within-Person
}

\section{Intention-Behavior Relations}

Jennifer Inauen, Ph.D.

Columbia University

Patrick E. Shrout, Ph.D.

New York University

Niall Bolger, Ph.D.

Columbia University

Gertraud Stadler, Ph.D.

Columbia University

Urte Scholz, Ph.D.

University of Zurich

Correspondence concerning this article should be addressed to Jennifer Inauen, Department of Psychology, Columbia University, 219 Schermerhorn Ext, 1190 Amsterdam Avenue, New York, NY 10027, phone: +1 212854 0127, email: ji2230@ columbia.edu

\section{Acknowledgement}

This work was supported by the University of Konstanz, Germany. The first author was supported by a fellowship of the Swiss National Science Foundation (Fellowship

$$
\text { P2ZHP1_155103). }
$$




\begin{abstract}
Background: Despite their good intentions, people often do not eat healthily. This is known as the intention-behavior gap. Although the intention-behavior relationship is theorized as a within-person process, most evidence is based on between-person differences.

Purpose: To investigate the within-person intention-behavior association for unhealthy snack consumption.

Methods: Young adults $(N=45)$ participated in an intensive longitudinal study. They reported intentions and snack consumption five times daily for seven days ( $n=1068$ observations analyzed).
\end{abstract}

Results: A within-person unit difference in intentions was associated with a halving of the number of unhealthy snacks consumed in the following three hours ( $\left.\mathrm{CI}_{95} 27-70 \%\right)$. Betweenperson differences in average intentions did not predict unhealthy snack consumption.

Conclusions: Consistent with theory, the intention-behavior relation for healthy eating is best understood as a within-person process. Interventions to reduce unhealthy snacking should target times of day when intentions are weakest.

Keywords: intention-behavior gap; intraindividual and interindividual associations; health behavior; snack consumption; ecological momentary assessment 
Unhealthy eating is one of the major risk factors for cardiovascular disease, diabetes, and obesity [1]. One source of unhealthy food intake is between-meal snack consumption, which has been linked to increased energy intake [2]. Because unhealthy snacks are ever more present in daily life, people who have goals to maintain a healthy weight are increasingly faced with the task of regulating their eating behavior. However, even though many people intend to avoid the consumption of unhealthy snacks, previous research suggests they often fail to enact their intentions [3-7]. This phenomenon of a weak intentionbehavior link is commonly known as the intention-behavior gap [6].

Behavioral intentions have been defined as “... people’s decisions to perform particular actions.” [6, p. 2]. They are a core behavioral predictor of well-researched health behavior theories, including the Theory of Planned Behavior [8], and the Health Action Process Approach [9]. Intentions are likely to consist of a relatively stable, trait-like component as well as a state-like component that fluctuates over time. For example, a person might generally intend to eat healthy food, but change this usual intention when offered a rich cake at her niece's birthday party. In this paper, we will refer to the trait-like component as the between-person intention; it indicates between-person differences in intentions overall. The state-like component is referred to as the within-person intention; it reflects persons' variation around their overall intention. It is known that between-person associations can differ substantially from those at the within-person level in size or even direction [10]. It is an open question whether this is the case for intention-behavior relations. Behavioral theories propose strong within-person intention-behavior links. They make no assumption about the nature of between-person intention-behavior relations, i.e., how the differences in persons' overall intentions are related to the consumption of unhealthy snacks.

Prior studies have focused on investigating intention-behavior relations by comparing persons with differing intention strengths at a particular point in time, in a specific situation. Such measures are composites of between-person and within-person intentions, but these are 
masked and cannot be separated by such research designs. For example, Conner et al. [11] asked hospital workers about their intentions to "eat a low-fat diet in the future" (p. 481) and correlated this with retrospectively reported behavior three months later. The intention measure was likely influenced by both the between-person intention (i.e., the general inclination to eat a low-fat diet), and a within-person deviation from the between-person intention (e.g., a situational increase in intention, because of having weighed one pound more than usual on the day of the study). Meta-analyses of these correlational intention-behavior studies found that intentions account for approximately 20-30 percent of variance in behavior $[3,6]$. These findings were supported by Webb and Sheeran's [7] meta-analysis that only included experimental studies. Their review found that moderate to strong changes in intentions foster weak to moderate changes in behavior (see also, [5]). This experimental evidence allows for confident conclusions about the causal relation of intentions and behavior. However, it provides little insight into within-person processes in daily life, they do not allow one to distinguish the between-person intention from within-person variability of intentions that first studies in other behavioral domains found in everyday life $[12,13]$.

The within-person intention-behavior relationship has received less attention in previous research. Those papers that have discussed within-person processes have tended to focus on the stability of intentions over time, which is hypothesized to be an indicator of intention strength, and hence should moderate the intention-behavior relationship such that more stable intentions lead to better translation of intentions into actions [14]. Several studies on intention stability have shown support for this hypothesis [15-17]. However, these studies typically operationalize intention stability by taking into account only two time points [18], which are rather imprecise measures of stability. What is more, summarizing within-person variability with a single person-specific number, rather than studying the dynamic relations of intentions to subsequent behaviors, cannot reveal the time-related within-person processes. 
To disentangle and simultaneously test between-person and within-person intentionbehavior relations in an ecologically valid setting, we conducted an intensive longitudinal study in everyday life [19]. In line with behavior theories' assumptions, we hypothesized that substantial positive intention-behavior associations can be found at the within-person level. Furthermore, we explored whether between-person intention-behavior relations differ from within-person intention-behavior relations. This represents the first simultaneous investigation of intention-behavior relations as a within-person process and from a betweenperson differences perspective.

\section{Method}

We conducted an intensive longitudinal study with five daily, time-based ecological momentary assessments [20] on seven consecutive days in Germany in May and June 2013. Diaries are the method of choice to answer this research question. However, possible diary reactivity, i.e., that completing a diary may influence the phenomenon of interest, could potentially endanger the validity of results obtained with this research method [21]. Tobias and Inauen [22] therefore recommend estimating the obtrusiveness of a diary in any given study. In this study, we estimated the presence of diary reactivity by comparing the diary group (diary+panel) to a control group (panel-only) regarding their unhealthy snack consumption and behavioral intentions on the day before and after the diary period.

\section{Participants}

We focused on persons who face a self-regulation dilemma with regards to unhealthy snack consumption, i.e., persons were eligible if they asserted that they generally intended to avoid unhealthy snacks, and admitted to occasionally eating unhealthy snacks. Furthermore, participants had to have a routine of eating two or more regular meals a day, have access to a smartphone during the study, be fluent in reading and writing German, and not be on vacation 
during the study. For a chart of the participant flow through the study, see Electronic Supplementary Material 1.

We a priori aimed at recruiting 50 participants for each condition (diary+panel and panel-only) based on issues of feasibility to recruit the sample in a short time frame (this study was conducted as part of a University course). Post-hoc power analyses revealed that the ultimately recruited sample of 45 (diary+panel) and 44 participants (panel-only) provided $80 \%$ power to detect a difference of .11 in snack consumption, which would be considered to be a medium effect size $(d=0.61)$.

In total, 89 young adults participated (diary+panel: $n=45$; panel-only: $n=44$ ). Five of them did not attend the second lab assessment, but they completed the questionnaire online. Participants in the diary+panel condition were asked to fill in the diaries five times per day on seven days. Of the resulting 1575 possible observations, 137 (8.7\%) were missing. Fifty-nine (66.3\%) of the participants were female. They were 18 to 29 years old $(M=22.0, S D=2.3)$, with a mean Body Mass Index (BMI) of $22.9(S D=2.5)$.

\section{Measures}

All measures were presented in German. English translations are described below (the original items are available at request). For comparison purposes, the same questions for snack consumption and intentions were included in the diary and the panel.

\section{Unhealthy snack consumption.}

Eating occurs frequently and recollections of it are prone to retrospective bias [23]. To prevent this, we chose frequent assessments at 3-hour intervals during the day, and a 12-hour interval during the night. In the present study, a snack was defined as any food and sweetened beverages consumed between main meals. Unhealthy snacks comprised the nine non-core foods adapted from Kelly, Smith, King, Flood, and Bauman [24], e.g. candy, cakes, and sugared drinks. Participants were given the detailed definition of healthy and unhealthy 
snacks based on the Kelly et al. distinction of core and non-core foods prior to the survey. Also, they were able to access this definition at any time during the diary surveys via a link on every page of the electronic questionnaire.

To facilitate remembering the snacks eaten between diary entries, participants were guided with the following questions. Firstly, participants were asked how often they had snacked since the last questionnaire (or on a typical day in the last seven days in the panel survey). If they had snacked at least once, they were asked to check the healthy (e.g., vegetables, breads) and unhealthy foods (e.g., candy, cakes, sugared drinks; 24) they had snacked since the last questionnaire. Also, for each checked category (e.g., candy), they were asked to write down the exact snacks they had consumed. The final behavioral measure was the count of all unhealthy snacks consumed. Participants were also asked to indicate on a 5point scale how large the portion of unhealthy snacks had been $(-2=$ much smaller than my usual portion to $2=$ much larger than my usual portion). But because the portion-size weighted measure yielded the same results as the unweighted measure, we only report the unweighted measure, as it is more straightforward to interpret.

\section{Intentions to avoid unhealthy snacking.}

Intentions were assessed with a typical measure of intention strength, in line with the Theory of Planned Behavior [8] and previous research on unhealthy snacking [25, 26]. The time reference captured momentary intention strength: “At this moment, to what extent do you intend to avoid unhealthy snacks?" The response options ranged from 1 "at this moment, not at all" to 9 "at this moment, very strongly". To allow for meaningful interpretation of the coefficient in the regression analysis, the scale was transformed into a range of 0 to 1 . We chose a 1-item measure of intention to avoid participation fatigue and ensure continued participation across the high-frequent assessments. Intentions are often operationalized with one or two items (e.g., [25, 27]). Our measure is derived from previous research [25, 26], and has high content validity, wherefore this 1-item measure can be considered appropriate (cf. 
[28]). As a further test, we computed correlations of the intention measure with related constructs. The results presented in Electronic Supplementary Material 2 show the expected correlations of intention with related constructs indicating construct validity.

\section{Dietary restraint}

Dietary restraint has been shown to influence snacking behavior [29]. Therefore, we included it as a covariate, using the restraint subscale of the German translation of the Dutch Eating Behavior Questionnaire (DEBQ; [29, 30]).

\section{Procedures}

This study was approved by the local Institutional Review Board. E-mail invitations were sent to all students of two universities. The incentive for study participation was taking part in a lottery of four vouchers worth EUR 150 each (app. \$ 167) for Amazon.com, or study participation credit.

The e-mail invitation contained a link to a brief online questionnaire that assessed eligibility criteria. Persons who met the criteria were then contacted by the experimenters. Prior to the phone call, the experimenters randomized the respective participant to one of the two conditions (diary+panel vs. panel-only) by entering the person's participant identification code into the next empty container of a block randomization list (each block had six containers). This list was previously created by a research assistant not involved in this study using random number generation. During the phone call, potential participants of the diary+panel condition were informed that they would receive EUR 20 (app. \$22) for this task (or equivalent additional course credit), in addition to participating in the lottery. After obtaining oral informed consent, appointments at the lab were scheduled.

At T1, after obtaining written informed consent, participants answered the T1 online questionnaire. After completing the questionnaire, participants' weight and height was measured, and the appointment ended for panel-only participants. Diary+panel participants 
received additional instructions for the diary: They would receive text messages containing the link to the online survey at $11 \mathrm{am}, 2 \mathrm{pm}, 5 \mathrm{pm}, 8 \mathrm{pm}$, and $11 \mathrm{pm}$ for the next seven days, starting the next day.

At $\mathrm{T} 2$, the eighth day after T1, all participants returned to the lab and again answered the online questionnaire. Moreover, their weight was measured, they were debriefed and received their course credit or were entered in the lottery.

\section{Data Analysis}

The key outcome, number of unhealthy snacks consumed, had a skewed distribution, and so we used a generalized linear model approach that specified a negative binomial distribution and a log link function [31]. The natural logarithm of number of snacks consumed was specified to be a linear function of intentions in the period prior to the snacking report (between person average intentions and the within-person deviation of intentions) and indicators of time of day and ordinal day within the study. To take into account the dependence of the observations within person and over time, we used generalized estimation equation (GEE) methods $[32,33]$. In comparison to multilevel modeling, GEE does not estimate random effects, but adjusts the model coefficients and standard errors for the interdependence between measurements. GEEs are particularly useful when modeling non-normally distributed outcomes, as for these, the specification of random effects using multilevel modeling can be difficult. We specified the working correlation structure as exchangeable. The effect sizes are rate ratios $(R R)$. They are interpreted as the percentage increase (values $>1$ ) or decrease (values $<1$ ) in snack consumption for a unit increase in the predictor [34]. All analyses were conducted with IBM SPSS Statistics 22.0.

To keep lags between intentions and snack consumption consistent at three hours, the $11 \mathrm{am}$ snack consumption measures (and the corresponding $11 \mathrm{pm}$ intentions) were removed prior to analyses. The between-person intention was computed by calculating the average 
intention for each person across all time points of the diary (except $11 \mathrm{pm}$ intentions). This variable was grand-mean centered to allow for a meaningful interpretation of the intercept. The within-person intention was calculated by centering intentions at the person mean, resulting in intrapersonal fluctuations around the person-specific mean across the study period. In the analyses, within-person intentions at a particular time predicted snack consumption at the next period. Two different time variables were included in the model: a linear time trend across all the time points of the study, coded from $0=$ first diary to $1=34^{\text {th }}$ diary, and time of day as a factor, coded $1=2 \mathrm{pm}, 2=5 \mathrm{pm}, 3=8 \mathrm{pm}$ and $4=11 \mathrm{pm}$. To test the hypothesis that the between-person intention-behavior association differs from within-person associations, the regression model was computed again, this time contrasting the betweenperson intention predictor to the original intention variable (cf. [35]). Finally, as a sensitivity analysis, the model was re-run, adjusting for age, gender, Body Mass Index (BMI), dietary restraint, past behavior (from previous period, or the same period the previous day), weekend, and the time lag of answering the surveys.

\section{Results}

\section{Intention-Behavior Relations}

The average intention to avoid unhealthy snacking assessed in the diary was rather strong: $0.64(S D=0.32)$. The intra-class correlations for intentions was 0.50 , which means that $50 \%$ of the variance was attributable to the person, and $50 \%$ was attributable to fluctuations within persons. Snack consumption ranged from 0-4 snacks consumed during the 3-hour reporting periods. However, the distribution was highly skewed; participants reported that they had consumed no unhealthy snacks in $77 \%$ of the diary entries. The intra-class correlation for snack consumption was 0.06 , indicating that almost all variability was within persons. 
The negative binomial generalized estimating equation model reported in Table 1 tested between-person and within-person effects of intentions to avoid unhealthy snacks on subsequent snack consumption. The model adjusted for changes in snack consumption over the study period and fluctuations of snack consumption during the day. The results indicated that participants significantly decreased their unhealthy snack consumption over the study period. Also, participants consumed significantly fewer unhealthy snacks between $11 \mathrm{am}-2 \mathrm{pm}$ and 5-8pm compared to the $8-11 \mathrm{pm}$ period. Over and above these temporal influences, between-person differences in persons' average intentions were weakly positively but not significantly associated with the average number of unhealthy snacks consumed. In line with our hypothesis, however, within-person fluctuations in intentions were significantly negatively related to subsequent unhealthy snack consumption. The rate ratio $(R R)$ indicates that when participants had one unit stronger intentions than usual, they subsequently ate $53 \%$ $\left(\mathrm{CI}_{95}=27 \%\right.$ to $\left.70 \%\right)$ fewer unhealthy snacks than at times with average intentions. This also means that persons with weaker intentions than usual subsequently consumed more unhealthy snacks. Regarding our second aim to investigate the differences in the between-person and within-person intention-behavior relations, a second model indicated that fluctuations in intentions to avoid unhealthy snacks were significantly more negatively related to subsequent snack consumption than between-person differences in average intentions ( $B=1.07, S E=.47$, $p=.023)$.

A sensitivity analysis of the model revealed no significant effects of age, gender, BMI, dietary restraint, past behavior, weekend and time lag between filling in the surveys on unhealthy snack consumption. The model results remained substantively unchanged after inclusion of these covariates. Figure 1 depicts a visualization of the model results. Predicted unhealthy snack consumption is displayed as a function of prior within-person intentions, adjusted for study period, time of day, and between-person differences in intentions. 


\section{Estimating Diary Reactivity}

To estimate whether the diary had affected snack consumption and intentions, we compared a panel-only group to the diary+panel group. Indicating that randomization was successful, we found no baseline differences between the diary+panel and the panel-only group regarding gender, age, BMI, intentions, or snack consumption (see also Electronic Supplementary Material 3). The analyses of group (diary+panel vs. panel-only) x time (T1 vs. T2) indicated that completing the diary in addition to the panel assessments had no effects on participants' snack consumption (Wald $[2]=0.35, p=0.839)$ or intentions $(F[1,87]<0.01$, $p=0.969$ ). There was a small main effect for time on snack consumption, however: Over time, unhealthy snack consumption decreased marginally, regardless of condition ( $\operatorname{Wald}[1]=4.28$, $p=0.039)$.

\section{Discussion}

This study is the first to investigate intention-behavior relations as a within-person process in addition to the between-person differences perspective. At the within-person level, in line with the assumptions of many health behavior theories (e.g., $[8,9])$, the results indicate strong intention-behavior associations: At times with stronger intentions, people ate 53 percent fewer unhealthy snacks than at times with average intentions. At the betweenperson level, no significant intention-behavior relations were found, supporting previous findings of a gap between intentions and behavior [5-7]. A statistical comparison of the between-person and within-person intention-behavior effects revealed that the within-person intention-behavior relations were stronger than the between-person ones.

The finding that within-person fluctuations in intentions predict later snack consumption confirms the importance of intention as a behavioral predictor as proposed by behavioral theories such as the Theory of Planned Behavior [8]. For the past decade, intentions have been conceptualized as a necessary but often insufficient prerequisite for 
behavior change. In contrast, our study shows that fluctuations in intentions can be meaningful to regulate behavior in daily life and can therefore also be considered a selfregulatory resource. As such, our results extend previous views on intention fluctuations that proposed that intention stability is a feature of intention strength, and variability therefore a weakness $[14,18]$. Rather, this depends on the direction of change.

Our results also show that even persons who are generally motivated can be sensitive to situational changes in intentions. This calls into question a categorical "stage" distinction of intenders and non-intenders of health behaviors. This also has important implications for health behavior change interventions. Highly motivated persons' intentions should not be presumed a given. Even motivated persons may benefit from reminding of their intentions in the moments of temptation. This may be achieved by real-time interventions in people's daily lives [36], for example, by subliminal goal priming [37], or reminding of personal goals via text messaging.

In our data, the within-person association between intention and behavior was much stronger than the between-person association. We did not find the small to moderate betweenperson intention-behavior associations reported in previous studies [3, 6]. Instead, our results imply that differences in unhealthy snack consumption cannot be explained by differences in persons' general motivation to avoid unhealthy snacking. Rather, increases or decreases in intentions during their daily lives seem crucial. To our knowledge, our study is the first to show the actual between-person effect, separated from the state-like influence on intentions (i.e., the within-person effect). This was made possible by the diary design. Previous studies employed single-time measures that mix trait-like between-person and state-like withinperson variances in intentions. Therefore, our result is not directly comparable to previous studies' findings of small-to-moderate between-person intention-behavior associations. Conclusions about the between-person intention-behavior relations should be drawn tentatively, however. First, we studied persons who were experiencing a self-regulation 
challenge. Therefore, future studies should investigate whether our findings also extend to persons who generally have no intention to avoid unhealthy snacks, or who never eat such snacks at all. But even with this sample restriction, there was variation in between-person intentions to avoid unhealthy snacks. By examining the relation of these between-person intentions and snack consumption, we were able to reflect the within-person association we found: Persons who generally had strong intentions to avoid unhealthy snacks were just as susceptible to dysregulation as others when they momentarily dropped their intention to avoid unhealthy snacks. Second, we did not consider the temporal order of between-person intention-behavior relations as we did for the within-person ones. For the between-person intention-behavior relations this was not meaningful in our study design, as we would not expect this trait-like process to change over short periods of time. Future studies should include larger time intervals to investigate lagged effects of between-person intentionbehavior relations.

This is the first study to disentangle between-person and within-person intentionbehavior associations. The intensive longitudinal design allowed the investigation of withinperson processes in people's daily lives, during several days. Thus, a particular strength of this study is its ecological validity. Furthermore, assessing people's snack consumption and intentions multiple times during the day enabled us to include the time of day in the model of intention-behavior relations. Despite the high-frequent assessments, no additional measurement reactivity was observed compared to the panel survey.

The non-causal research design of the study is a limitation. Although time-sequencing ensured that prior intentions predicted later snack consumption, the third variable problem [38] cannot be ruled out. Results from meta-analyses of experimental studies on intentionbehavior relations $[5,7]$ encourage causal interpretation. Nevertheless, the next step in investigating within-person intention-behavior relations is to strengthen people's intentions in everyday life using real-time interventions [36]. Another possible criticism is that our sample 
was limited to young adults. In the case of unhealthy snacking, however, we argue that studying young adults is meaningful, because they have been shown particularly prone to unhealthy snacking [39]. Snack consumption was self-reported. Even though the design and procedure minimized retrospective bias, it is possible that participants' reporting of their snack consumption exhibited social desirability. As greater consistency has been found for intentions and objectively measured behavior [7], our results may have underestimated the strength of intention-behavior associations. Also, greater intention-behavior consistency may be found if the time reference in the intention measure paralleled the interval of the retrospective snack consumption report, compared to the momentary intention measure used in our study.

This research indicates that situational changes in intentions may strongly impact subsequent behavior. Still, intentions did not explain all behavioral variance. Future studies should therefore investigate additional factors at the within-person level, such as action control [40], implementation intentions [41, 42], and habitual processes [43, 44].

In conclusion, this research provided support for behavioral theories' assumption of the importance of intentions to predict behavior at the within-person level. More generally, the significance of considering both between-person differences and within-person processes when aiming at explaining health behaviors was stressed, as these may differ. Enhancing people's intentions to avoid eating unhealthy snacks in the moments of temptation holds promise to support people's self-regulation of eating behavior in their daily lives.

\section{References}

1. World Health Organization. Diet, nutrition, and the prevention of chronic diseases: WHO Technical Report Series, 916; 2003. 
2. Graaf $\mathrm{C}$ de. Effects of snacks on energy intake: An evolutionary perspective. Appetite. 2006; 47: 18-23.

3. Armitage CJ, Conner M. Efficacy of the theory of planned behaviour: A meta-analytic review. British Journal of Social Psychology. 2001; 40: 471-499.

4. Conner M, Sparks P. The theory of planned behaviour and health behaviours. In: Conner M, Norman P, eds. Predicting health behaviour: Research and practice with social cognition models. 2nd ed. Buckingham, England: Open University Press; 2005: 170-222.

5. Rhodes RE, Dickau L. Experimental evidence for the intention-behavior relationship in the physical activity domain: A meta-analysis. Health Psychology. 2012; 31: 724-727.

6. Sheeran P. Intention-behavior relations: A conceptual and empirical review. European Review of Social Psychology. 2002; 12: 1-36.

7. Webb TL, Sheeran P. Does changing behavioral intentions engender behavior change? A meta-analysis of the experimental evidence. Psychological Bulletin. 2006; 132: 249-286.

8. Ajzen I. The theory of planned behavior. Organizational Behavior and Human Decision Processes. 1991; 50: 179-211.

9. Schwarzer R. Modeling health behavior change: How to predict and modify the adoption and maintenance of health behaviors. Applied Psychology: An International Review. 2008; 57: 1-29.

10. Hamaker EL. Why researchers should think "within-person": A paradigmatic rationale. In: Mehl MR, Conner TS, eds. Handbook of research methods for studying daily life. New York: Guildford Press; 2012: 43-61.

11. Conner M, Sheeran P, Norman P, Armitage CJ. Temporal stability as a moderator of relationships in the theory of planned behaviour. British Journal of Social Psychology. 2000; 39: 469-493.

12. Conroy DE, Maher JP, Elavsky S, Hyde AL, Doerksen SE. Sedentary behavior as a daily process regulated by habits and intentions. Health Psychology. 2013; 32: 1149-1157. 
13. Kiene SM, Tennen H, Armeli S. Today I'll use a condom, but who knows about tomorrow: A daily process study of variability in predictors of condom use. Health Psychology. 2008; 27: 463-472.

14. Sheeran P, Orbell S, Trafimow D. Does the temporal stability of behavioral intentions moderate intention-behavior and past behavior-future behavior relations? Personality and Social Psychology Bulletin. 1999; 25: 724-734.

15. Conner M, Godin G. Temporal stability of behavioural intention as a moderator of intention-behaviour relationships. Psychology \& Health. 2007; 22: 875-897.

16. Cooke R, Sheeran P. Moderation of cognition-intention and cognition-behaviour relations: A meta-analysis of properties of variables from the theory of planned behaviour. Br. J. Soc. Psychol. 2004; 43: 159-186.

17. Sheeran P, Abraham C. Mediators and moderators: Temporal stability of intention and the intention-behavior relation. Pers Soc Psychol Bull. 2003; 29: 205-215.

18. Kellar I, Hankins M. Intention stability: Literature and methodological review. Revue Européenne de Psychologie Appliquée/European Review of Applied Psychology. 2013; 63: $363-373$.

19. Bolger N, Laurenceau J. Intensive longitudinal methods: An introduction to diary and experience sampling research. New York: Guilford Press; 2013.

20. Shiffman S. Dynamic influences on smoking replapse process. Journal of Personality. 2005; 73: 1715-1748.

21. Stone AA, Broderick JE, Schwartz JE, Shiffman S, Litcher-Kelly L, Calvanese P. Intensive momentary reporting of pain with an electronic diary: Reactivity, compliance, and patient satisfaction. Pain. 2003; 104: 343-351.

22. Tobias R, Inauen J. Gathering time-series data for evaluating behavior-change campaigns in developing countries: Reactivity of diaries and interviews. Evaluation Review. 2010; 34: $367-390$. 
23. Schwarz N. Retrospective and concurrent self-reports: The rationale for real-time data capture. The science of real-time data capture: Self-reports in health research. 2007: 1126.

24. Kelly B, Smith B, King L, Flood V, Bauman A. Television food advertising to children: The extent and nature of exposure. Public Health Nutrition. 2007; 10: 1234-1240.

25. Gardner B, Corbridge S, McGowan L. Do habits always override intentions? Pitting unhealthy snacking habits against snack-avoidance intentions. BMC psychology. 2015; 3: 8.

26. Allan JL, Johnston M, Campbell N. Unintentional eating. What determines goalincongruent chocolate consumption? Appetite. 2010; 54: 422-425.

27. Scholz U, Nagy G, Göhner W, Luszczynska A, Kliegel M. Changes in self-regulatory cognitions as predictors of changes in smoking and nutrition behaviour. Psychology and Health. 2009; 24: 545-561.

28. Rossiter JR. Marketing measurement revolution: The C-OAR-SE method and why it must replace psychometrics. European Journal of Marketing. 2011; 45: 1561-1588.

29. van Strien T, Frijters JER, Bergers G, Defares PB. The Dutch Eating Behavior Questionnaire (DEBQ) for assessment of restrained, emotional, and external eating behavior. International Journal of Eating Disorders. 1986; 5: 295-315.

30. Grunert SC. Ein Inventar zur Erfassung von Selbstaussagen zum Ernährungsverhalten. Diagnostica. 1989; 35: 167-179.

31. Gardner W, Mulvey EP, Shaw EC. Regression analyses of counts and rates: Poisson, overdispersed Poisson, and negative binomial models. Psychological Bulletin. 1995; 118: 392.

32. Liang K, Zeger SL. Longitudinal data analysis using generalized linear models. Biometrika. 1986; 73: 13-22. 
33. Hardin JW, Hilbe JM. Generalized estimating equations. Boca Raton, FL: CRC Press; 2013.

34. Atkins DC, Baldwin SA, Zheng C, Gallop RJ, Neighbors C. A tutorial on count regression and zero-altered count models for longitudinal substance use data. Psychology of Addictive Behaviors. 2013; 27: 166-177.

35. Snijders TAB, Bosker RJ. Multilevel analysis: An introduction to basic and advanced multilevel modeling. London: Sage; 1999.

36. Heron KE, Smyth JM. Ecological momentary interventions: Incorporating mobile technology into psychosocial and health behaviour treatments. Br J Health Psychol. 2010; 15: 1-39.

37. Papies EK, Hamstra P. Goal priming and eating behavior: Enhancing self-regulation by environmental cues. Health Psychology. 2010; 29: 384-388.

38. Mauro R. Understanding L.O.V.E. (left out variables error): A method for estimating the effects of omitted variables. Psychological Bulletin. 1990; 108: 314-329.

39. Papadaki A, Hondros G, Scott JA, Kapsokefalou M. Eating habits of university students living at, or away from home in Greece. Appetite. 2007; 49: 169-176.

40. Sniehotta FF, Scholz U, Schwarzer R. Bridging the intention-behaviour gap: Planning, self-efficacy, and action control in the adoption and maintenance of physical exercise. Psychology \& Health. 2005; 20: 143-160.

41. O’Connor DB, Armitage CJ, Ferguson E. Randomized test of an implementation intention-based tool to reduce stress-induced eating. Annals of Behavioral Medicine. 2014: 1-13.

42. Gollwitzer PM. Implementation intentions: Strong effects of simple plans. American Psychologist. 1999; 54: 493-503.

43. Rothman AJ, Sheeran P, Wood W. Reflective and automatic processes in the initiation and maintenance of dietary change. Annals of Behavioral Medicine. 2009; 38: 4-17. 
44. Verplanken B, Aarts H, van Knippenberg A, Moonen A. Habit versus planned behaviour: A field experiment. British Journal of Social Psychology. 1998; 37: 111-128. 
Table 1

Parameter Estimates for Negative Binomial Generalized Estimating Equations Model of the Number of Unhealthy Snacks Consumed as a Function of Time and Intentions to Avoid Unhealthy Snacking

\section{$\mathrm{CI}_{95}$ for $R R$}

\begin{tabular}{|c|c|c|c|c|c|c|}
\hline Fixed effects & $B$ & $S E$ & $p$ & $R R$ & Lower & Upper \\
\hline Intercept & -0.70 & 0.16 & $<0.001$ & 0.50 & 0.37 & 0.68 \\
\hline Time (study period) & -0.53 & 0.19 & 0.004 & 0.59 & 0.41 & 0.85 \\
\hline Daytime $-2 \mathrm{pm}^{\mathrm{a}}$ & -0.47 & 0.14 & 0.001 & 0.63 & 0.48 & 0.83 \\
\hline Daytime $-5 \mathrm{pm}^{\mathrm{a}}$ & -0.14 & 0.16 & 0.383 & 0.87 & 0.63 & 1.19 \\
\hline Daytime $-8 \mathrm{pm}^{\mathrm{a}}$ & -0.43 & 0.15 & 0.005 & 0.65 & 0.48 & 0.88 \\
\hline Between intention & 0.30 & 0.38 & 0.425 & 1.36 & 0.64 & 2.86 \\
\hline Within intention & -0.77 & 0.23 & 0.001 & 0.47 & 0.30 & 0.73 \\
\hline
\end{tabular}

Note. $N=45$ persons, 27 measurement times, 1068 observations. Scale parameter: $0.675 .^{\text {a }}$

Relative to $11 \mathrm{pm} . R R=$ Rate ratio. 


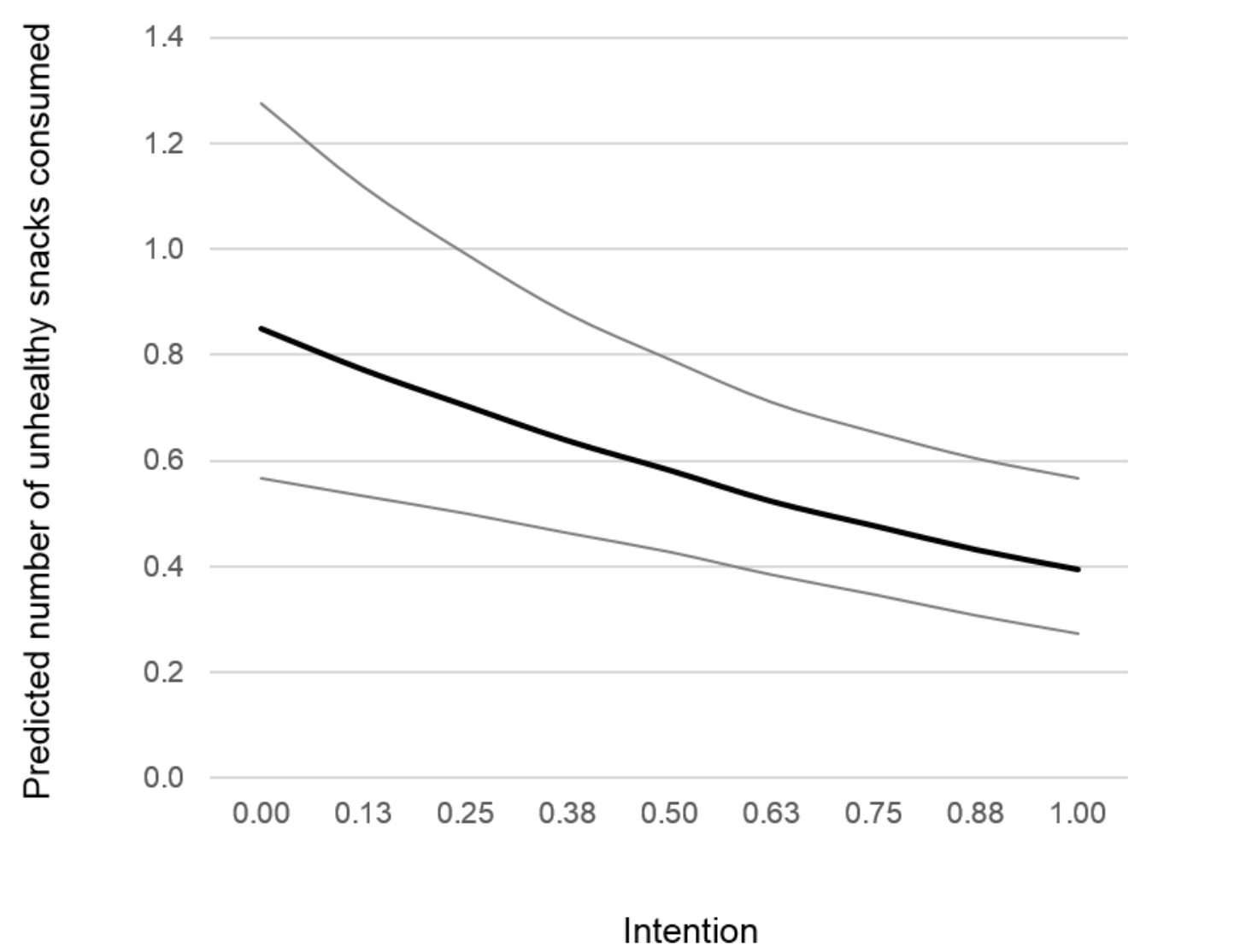

Figure 1. Predicted unhealthy snack consumption as a function of prior within-person intentions, adjusted for study period, time of day, and between-person differences in intentions. The thick line represents the average effect. The thin lines reflect the uncertainty (+/- 2 times the standard error). 